\title{
Trigonometric Interpolation and Curve-Fitting
}

\author{
By A. C. R. Newbery
}

\begin{abstract}
Some algorithms are introduced, whereby a function defined on an arbitrarily spaced set of abscissas may be interpolated or approximated by trigonometric or hyperbolic polynomials. The interpolation may be ordinary or osculatory. Least squares approximation is included; the approximant may be a pure sine series or a cosine series or a balanced trigonometric or hyperbolic polynomial. An application to a periodicity-search is described.
\end{abstract}

An extensive set of algorithms is available for functional approximation and interpolation in terms of polynomials. The present article develops some corresponding algorithms for nonpolynomial approximants. The classes of approximant (interpolant) considered are sine polynomial, cosine polynomial, balanced trigonometric polynomial and their analogs in terms of hyperbolic functions. The classes of approximation considered are interpolation on ordinates, osculatory and hyperosculatory interpolation, weighted least-squares approximation, weighted least-squares approximation subject to some ordinate and derivative constraints.

Trigonometric Analogs of Lagrange and Hermite Interpolation. Lagrangian and Hermitian interpolation in terms of sine polynomials were dealt with in [1], and the adaptation to cosine polynomials is straightforward. For Lagrangian interpolation in terms of balanced trigonometric polynomials there is a classical algorithm [2, p. 38], but we wish to develop an alternative which has some advantages with respect to economy and ease of generalization.

Let there be $N$ points $\left(x_{i}, f_{i}\right), i=1, \cdots N$; let all the abscissas $x_{i}$ be distinct and strictly within an interval $I$. It is required to construct a function

$$
y(x) \equiv \sum_{r=1}^{p} s_{r} \sin r \omega x+\sum_{r=0}^{Q} c_{r} \cos r \omega x
$$

such that $y\left(x_{i}\right)=f_{i}, p+q+1=N$ and $|p-q| \leqq 1$. (The latter condition defines a "balanced" trigonometric polynomial.) The parameter $\omega$ determines the frequency of the interpolant; there may be some restriction on values assigned to it. Let $\theta$ denote $\omega x$, let $\theta_{i}=\omega x_{i}$, and let $L_{i k}(\theta)$ denote a function of degree $(j, k)$, i.e., a function of the form $\sum_{r=1}^{i} s_{r}^{\prime} \sin r \theta+\sum_{r=0}^{k} c_{r}^{\prime} \cos r \theta$, such that $L_{i k}\left(\theta_{i}\right)=f_{i}$ for $i \leqq j+k+1$. Let $I^{\prime}$ be the range of $\theta$ induced by the requirement $x \in I$ and let $\Pi_{j i}(\theta)$ be a function of degree $(j, j)$ such that $\Pi_{i j}\left(\theta_{i}\right)=0$ for $i \leqq 2 j$; moreover, these $2 j$ zeros are the only zeros of $\Pi_{i j}$ in $I^{\prime}$. For consecutive $j$ we can now construct the functions $\Pi_{i j}$, which are unique to within a normalization factor. Starting with $\Pi_{00}(\theta) \equiv 1$, we may define for $j=0,1 \cdots \Pi_{i+1, i+1}=g_{j}(\theta) \Pi_{i}$, where $g_{i}(\theta)=\alpha_{i} \sin \theta+\beta_{i} \cos \theta-\gamma_{i}$.

Received December 29, 1969, revised April 20, 1970.

AMS 1969 subject classifications. Primary 6520, 6590; Secondary 4206, 4208, 4216.

Key words and phrases. Trigonometric interpolation, trigonometric curve-fitting, osculatory trigonometric interpolation, constrained trigonometric approximation, exponential approximation, periodicity detection, harmonic analysis. 
The coefficients of $g_{i}$ are chosen so that $g_{i}(\theta)$ shall be zero at $\theta_{2 j+1}, \theta_{2 j+2}$ and nowhere else in $I^{\prime}$. It may be verified that $g_{i}(\theta)$ has the required zeros if

$$
\begin{gathered}
\alpha_{i}=\cos \theta_{2 i+2}-\cos \theta_{2 i+1}, \quad \beta_{i}=\sin \theta_{2 i+1}-\sin \theta_{2 i+2}, \\
\gamma_{i}=\sin \left(\theta_{2 i+1}-\theta_{2 i+2}\right) .
\end{gathered}
$$

We can also guarantee that $g_{i}(\theta)$ has no other zeros in $I^{\prime}$ provided the width of $I^{\prime}$ is less than $2 \pi$. This can be seen by noting that alternate zeros of $g_{i}(\theta)$ (which are real by construction) occur at intervals of $2 \pi$. An interval of less than $2 \pi$ can, therefore, not contain more than two zeros of $g_{i}(\theta)$. Having shown that the functions $\Pi_{i j}(\theta)$ are constructible, we now construct for $j=0,1, \cdots$ the functions $L_{i j}(\theta)$ such that $L_{j i}\left(\theta_{i}\right)=f_{i}$ for $i \leqq 2 j+1$. These functions may be constructed by the recursion

$$
\begin{aligned}
L_{00}=f_{1}, \quad L_{i+1, i+1}=L_{i j} & +g_{i}^{*}(\theta) \Pi_{i j}(\theta), \\
& \text { where } g_{i}^{*}(\theta) \equiv \alpha_{i}^{*} \sin \theta+\beta_{i}^{*} \cos \theta-\gamma_{i}^{*} .
\end{aligned}
$$

From the definitions of $L_{i j}, \Pi_{i j}$ it follows that $L_{i+1, i+1}$ as defined in (2) must pass through the points $\left(\theta_{i}, f_{i}\right)$ for $i \leqq 2 j+3$. The coefficients $\alpha_{i}^{*}, \beta_{i}^{*}, \gamma_{i}^{*}$ must, therefore, be chosen so that $L_{i+1, j+1}$ will interpolate correctly at subscripts $i=2 j+1,2 j+2$, $2 j+3$. This is equivalent to

$$
\left[\begin{array}{lll}
\sin \theta_{2 i+1} & \cos \theta_{2 i+1} & -1 \\
\sin \theta_{2 j+2} & \cos \theta_{2 i+2} & -1 \\
\sin \theta_{2 i+3} & \cos \theta_{2 i+3} & -1
\end{array}\right]\left[\begin{array}{l}
\alpha_{i}^{*} \\
\beta_{i}^{*} \\
\gamma_{i}^{*}
\end{array}\right]=\left[\begin{array}{c}
0 \\
{\left[f_{2 i+2}-L_{i j}\left(\theta_{2 i+2}\right)\right] / \Pi_{i j}\left(\theta_{2 i+2}\right)} \\
{\left[f_{2 i+3}-L_{i j}\left(\theta_{2 i+3}\right)\right] / \Pi_{i j}\left(\theta_{2 i+3}\right)}
\end{array}\right] .
$$

It can be shown $[3$, p. 85$]$ that the determinant of the system is

$$
4 \sin \frac{\left(\theta_{2 i+2}-\theta_{2 i+1}\right)}{2} \sin \frac{\left(\theta_{2 i+3}-\theta_{2 i+1}\right)}{2} \sin \frac{\left(\theta_{2 i+3}-\theta_{2 i+2}\right)}{2} \text {. }
$$

The system, therefore, has to be solvable under our assumptions that the abscissas $\theta_{i}$ are distinct and within an interval of length $<2 \pi$. In these circumstances the recursion (2) enables us to produce the required trigonometric interpolant, provided the number of points $N$ is odd. If $N$ is even, let $N^{\prime}=(N-2) / 2$, let $L_{N^{\prime}, N^{\prime}}$ be the interpolant at $2 N^{\prime}+1=N-1$ points and construct $\Pi_{N^{\prime}, N^{\prime}}$ which has zeros at all abscissas subscripted 1 through $2 N^{\prime}=N-2$. We now attempt to define $L_{N^{\prime}+1, N^{\prime}} \equiv$ $L_{N^{\prime}, N^{\prime}}+g_{N^{\prime}}^{*}(\theta) \Pi_{N^{\prime}, N^{\prime}}(\theta)$. The linear equations defining the coefficients of $g_{N^{\prime}}^{*}$, resemble (3) in the first two rows with $N^{\prime}$ replacing $j$; however, the third constraint is the one which implies that $g_{N^{\prime}}^{*} \Pi_{N^{\prime} . N^{\prime}}$ should have no term involving $\cos \left(N^{\prime}+1\right) \theta$. Let $\Pi_{N^{\prime}, N^{\prime}}=$ $s_{N^{\prime}} \sin N^{\prime} \theta+c_{N^{\prime}} \cos N^{\prime} \theta+$ terms of lower frequency. The third constraint is then seen to be $-\alpha_{N}^{*}, s_{N^{\prime}}+\beta_{N}^{*}, c_{N^{\prime}}=0$. When we examine the matrix which defines $\alpha_{N^{\prime}}^{*}$, $\beta_{N}^{*}, \gamma_{N}^{*}$, we know already that the first two rows are independent under our hypotheses. Singularity could, therefore, occur if and only if the third row is a linear combination of the first two, or equivalently

$$
-s_{N^{\prime}} /\left(\sin \theta_{N}-\sin \theta_{N-1}\right)=c_{N^{\prime}} /\left(\cos \theta_{N}-\cos \theta_{N-1}\right) .
$$

In this event, $L_{N^{\prime}+1, N^{\prime}}$ will generally not be constructible. The same applies to $L_{N^{\prime}, N^{\prime}+1}$ in the event that $c_{N^{\prime}} /\left(\sin \theta_{N}-\sin \theta_{N-1}\right)=s_{N^{\prime}} /\left(\cos \theta_{N}-\cos \theta_{N-1}\right)$. In any case, it can be seen that at least one of the interpolants $L_{N^{\prime}+1, N^{\prime}}, L_{N^{\prime}, N^{\prime}+1}$ is constructible. 
Osculatory interpolation on $N$ points is a confluent case of ordinate interpolation on $2 N$ points. By defining $\theta_{N+r}=\theta_{r}$ for $r=1, \cdots, N$, we can construct $\Pi_{i j}$ by the same recursion as before for $j=1, \cdots, N-1$. For the earlier $j$-values $(j \leqq[N / 2])$, $\Pi_{i j}$ will satisfy homogeneous ordinate constraints as before; for later $j$-values $\Pi_{j i}$ will satisfy at least one repeated homogeneous ordinate constraint, i.e., it will satisfy a homogeneous derivative constraint. Thus, the algorithmic construction of $\Pi_{i i}$ is essentially unchanged from the previous paragraph, but the properties of the resulting function are modified. The Eqs. (3) will still hold if we add the proviso that any subscript in the range $[N+1,2 N]$ shall have $N$ subtracted from it. Moreover, when this occurs on the right side of (3) the quantity is replaced by its derivative. For example, if $2 j+2>N$, then the second equation of (3) would read:

$$
\alpha_{i}^{*} \sin \theta_{2 i+2-N}+\beta_{i}^{*} \cos \theta_{2 i+2-N}-\gamma_{i}^{*}=\left[f_{2 i+2-N}^{\prime}-L_{i j}^{\prime}\left(\theta_{2 i+2-N}\right)\right] / \Pi_{i j}^{\prime}\left(\theta_{2 i+2-N}\right) \text {. }
$$

The extension to hyperosculatory interpolation follows the same principle, although the details become harder to specify. We still generate a sequence of functions $\left\{\Pi_{i j}(\theta)\right\}$ satisfying $2 j$ homogeneous conditions; for low values of $j$ the conditions imply zeros of the function $\Pi_{i i}$ at specified nodes; when these conditions are all met, the subsequent conditions imply zero derivatives at a subset of these nodes; thereafter, they imply zero second derivatives at a subsubset of the nodes, etc. It is essential that the successive higher derivative constraints be built on in increasing order. For instance, one can construct $\Pi_{11}(\theta)$ to vanish at any two assigned points $\theta_{1}, \theta_{2}$ in $I^{\prime}$ or to vanish with its derivative at $\theta_{1}$, but it is generally impossible to construct a $\Pi_{11}$ of proper degree such that $\Pi_{11}^{\prime}(\theta)$ vanishes at $\theta_{1}, \theta_{2}$.

Least-Squares Approximation by Sine and Cosine Polynomials. The problem of determining a cosine polynomial which, in a weighted least-squares sense, best approximates a discrete function defined on arbitrarily spaced abscissas has been discussed by Oliveira-Pinto [4]. He showed that the problem could be reduced to the analogous problem for algebraic polynomials and solved by Forsythe's method [5]. We would like to observe that this problem and the corresponding sine series problem can also be treated by a direct method without any need for conversion into and out of algebraic form.

Let $N$ points $\left(x_{i}, f_{i}\right)$ and positive weights $W_{i}$ be specified. Let $\theta=\omega x$ as before, and let $\left\{\phi_{r}(\theta)\right\}$ be a set of orthogonal sine polynomials such that $\left(\phi_{r}, \phi_{s}\right) \equiv \sum_{i=1}^{N} \phi_{r}\left(\theta_{i}\right) \phi_{s}\left(\theta_{i}\right) W_{i}=0$ for $r \neq s$. These polynomials can be generated by the following recursion:

$$
\begin{aligned}
\phi_{1} & =\sin \theta, \quad \phi_{2}=\left(2 \cos \theta-a_{1}\right) \phi_{1}, \\
\phi_{k+1} & =\left(2 \cos \theta-a_{k}\right) \phi_{k}-b_{k} \phi_{k-1}, \quad \text { where } \\
b_{k} & =2\left(\cos \theta \phi_{k}, \phi_{k-1}\right) /\left(\phi_{k-1}, \phi_{k-1}\right), \quad \text { and } \\
a_{k} & =2\left(\cos \theta \phi_{k}, \phi_{k}\right) /\left(\phi_{k}, \phi_{k}\right) .
\end{aligned}
$$

The only event which could cause a stoppage is that for some $k\left(\phi_{k}, \phi_{k}\right)=0$. This in turn would imply that, for all $i, \phi_{k}\left(\theta_{i}\right)=0$, so that $\phi_{k}$ must be orthogonal to all functions with respect to our inner product. Although the function $\phi_{k}(\theta)$ is nontrivial by construction, it may generate a trivial $N$-vector on evaluation at the $N$ arguments $\theta_{i}$. 
In this case, we will call the function "degenerate". It follows from the method of construction that, if $\phi_{k}$ is degenerate, then all subsequent functions are also degenerate. It may be noted that $\phi_{N+1}$ is necessarily degenerate, because the contrary assumption would imply that there existed $N+1$ nontrivial vectors of dimension $N$ all mutually orthogonal with respect to our inner product. We now examine the question of whether and when "premature degeneration" can occur, i.e., in what circumstances can it happen that, for some $k<N+1, \phi_{k}$ is degenerate? We note that $\phi_{k}(\theta)$ can be written in the form $\left(1-z^{2}\right)^{1 / 2} P_{k-1}(z)$, where $z=\cos \theta$ and $P_{k-1}$ is an algebraic polynomial of degree $k-1$. There are at most $k+1 z$-values $z_{j}$ of magnitude $\leqq 1$ for which $\left(1-z^{2}\right)^{1 / 2} P_{k-1}(z)$ vanishes. Corresponding to each $z_{i}$ there may be several $\theta_{i}$ such that $\cos \theta_{i}=z_{j}$; however, the following observations may be made:

I. If $I^{\prime}$ is a range over which $\cos \theta$ is monotonic, then $\phi_{k}(\theta)$ vanishes at most $k$ times in $I^{\prime}$. Consequently, degeneration cannot occur earlier than at $\phi_{N}$. If we exclude values of $\theta_{i}$ for which $\sin \theta_{i}=0$ (which is a reasonable policy), then there cannot be a premature degeneration. This follows from the fact that for each $z_{i}$, of which there are at most $k-1$, there exists at most one $\theta_{i}$ such that $\cos \theta_{i}=z_{i}$. Since we excluded the possibility that $\sin \theta_{i}=0$ it follows that $\phi_{k}(\theta)$ cannot vanish more than $k-1$ times in $I^{\prime}$.

II. In many applications it will not be a reasonable policy to select $\omega$ in such a way that the length of $I^{\prime}$ exceeds $2 \pi$, and we now assume that the length is strictly less than $2 \pi$. In this situation we can guarantee that, for $k<N / 2, \phi_{k}$ is not degenerate. Furthermore, if for all distinct $i, j, \cos \theta_{i} \neq \cos \theta_{i}$ we can guarantee that $\phi_{N-2}$ is not degenerate. If we impose the additional (reasonable) restriction that $\sin \theta_{i} \neq 0$ then $\phi_{N}$ will not be degenerate. This may be argued as follows: There are at most $k-1$ values $z_{j}$; to each of these there will generally correspond two values of $\theta$ such that $\cos \theta=z_{i}$, but in view of our restrictions at most one of these can be a data point. Since we excluded the possibility that $\sin \theta_{i}=0$, it follows that $\phi_{k}$ vanishes at most $k-1$ times in $I^{\prime}$, and $\phi_{N}$ is, therefore, not degenerate.

Once the orthogonal functions $\left\{\phi_{r}\right\}$ have been constructed, the $n$th degree leastsquares approximation is given in the usual way by $f(\theta) \simeq \sum_{r=0}^{n} b_{r} \phi_{r}$, where $b_{r}=$ $\left(f(\theta), \phi_{r}\right) /\left(\phi_{r}, \phi_{r}\right)$. The occurrence of a premature degeneration does not imply that the algorithm will fail to produce the least-squares solution; it implies that the leastsquares error has reached a lower bound (which may or may not be zero) beyond which it cannot be further reduced regardless how many additional functions are adjoined to the space of the approximants. In short, it is a signal to stop augmenting the degree of the approximant.

If we wish to generate orthogonal cosine polynomials, we can use the same recursion (4) except that we initialize with $\phi_{0}=1, \phi_{1}=\cos \theta-(\cos \theta, 1) /(1,1)$. Having generated the orthogonal trigonometric polynomials, we find the $n$th degree trigonometric approximant to be $f(x) \simeq \sum_{r=0,1}^{n} t_{r} \phi_{r}(\omega x)$, where the lower summation limit is 0 for cosine and 1 for sine approximation, and

$$
t_{r}=\sum_{i=1}^{N} W_{i} f\left(x_{i}\right) \phi_{r}\left(\omega x_{i}\right) /\left(\phi_{r}, \phi_{r}\right) .
$$

Constrained Least-Squares Curve-Fitting. In the case of sine and cosine approximation we are able to incorporate exact constraints on ordinates and derivatives 
just as Klopfenstein did in the algebraic case [6]; by this we mean that certain constraints are to be met exactly, while others are to be satisfied in a least-squares sense. Let $L(\theta)$ be the sine (or cosine) polynomial of minimal degree satisfying all the exact constraints on ordinates and possibly on derivatives. Conditions under which $L$ is constructible and an algorithm for constructing it are given in [1]. Let $\Pi(\theta)$ be a cosine polynomial of minimal degree satisfying corresponding homogeneous conditions, e.g., if $L\left(\theta_{i}\right), L^{\prime}\left(\theta_{i}\right)$ are specified to be $f_{i}$ and $f_{i}^{\prime}$ then $\Pi\left(\theta_{i}\right), \Pi^{\prime}\left(\theta_{i}\right)$ have to be zero by construction. If we approximate $f(\theta)$ by $L(\theta)+\Pi(\theta) G(\theta)$, where $L$ and $G$ are trigonometric polynomials of the same kind (both sine or both cosine), then by construction we are meeting all the exact constraints; it only remains to determine $G(\theta)$ in such a manner as to match the remaining constraints optimally in a weighted least-squares sense. We have, therefore, to minimize the squared residual $E$ given by

$$
\begin{aligned}
E & =\sum_{i=1}^{N} W_{i}\left[L\left(\theta_{i}\right)+\Pi\left(\theta_{i}\right) G\left(\theta_{i}\right)-f\left(x_{i}\right)\right]^{2} \\
& =\sum W_{i} \Pi\left(\theta_{i}\right)\left[G\left(\theta_{i}\right)-\left(f\left(x_{i}\right)-L\left(\theta_{i}\right)\right) / \Pi\left(\theta_{i}\right)\right],
\end{aligned}
$$

where $\sum$ denotes summation over subscripts which are not exactly constrained. Assuming there is no vanishing denominator in the last expression (5) we now have reduced the constrained problem to a standard unconstrained problem of the kind considered above, with modified approximand and weight function. Two observations can be made concerning the case where a denominator in (5) vanishes. Firstly, if the problem is normalized in such a way that $i \neq j$ implies $\cos \theta_{i} \neq \cos \theta_{i}$ then there can be no vanishing denominator; the function $\Pi(\theta)$ would not vanish at data points other than those which are exactly constrained, and these are explicitly excluded from the summation. Secondly, in the event of a denominator vanishing, if we look at the limiting form, i.e., the second expression in (5), we find that at one abscissa $\theta_{i}, G\left(\theta_{i}\right)$ is multiplied by zero. Whatever choice of coefficients we may make for $G(\theta)$, our decision can therefore have no effect at that abscissa. If we delete that abscissa entirely, the deletion can have no effect on the optimal choice of coefficients. It will affect the value of $E$ in a determinable manner, but it will not affect the variation of $E$. It is the variation of $E$ and not the value which de nines the optimal coefficients of $G(\theta)$.

Least-Squares Approximation by a Balanced Trigonometric Series. Let $B_{p e}(\theta)$ denote a trigonometric polynomial of the form

$$
\sum_{r=0}^{p} s_{r} \sin r \theta+\sum_{r=0}^{Q} c_{r} \cos r \theta
$$

We shall need to construct an orthogonal sequence $B_{00}, B_{10}, B_{11}, B_{21}, B_{22}, \cdots, B_{k k}$, $B_{k+1, k} \cdots$. The inner product defining the orthogonality is

$$
\left(B_{p a}, B_{r s}\right) \equiv \sum_{i=1}^{N} W_{i} B_{p q}\left(\theta_{i}\right) B_{r s}\left(\theta_{i}\right) .
$$

Let it be assumed that we have constructed the orthogonal sequence as far as $B_{k k}$, and the normalization convention is that 


$$
\begin{aligned}
B_{k k} & =s_{k} \sin k \theta+\cos k \theta+\hat{s}_{k-1} \sin (k-1) \theta+\hat{c}_{k-1} \cos (k-1) \theta+\cdots \\
B_{k, k-1} & =\sin k \theta+\tilde{s}_{k-1} \sin (k-1) \theta+c_{k-1} \cos (k-1) \theta+\cdots .
\end{aligned}
$$

In order to construct $B_{k+1, k}, B_{k+1, k+1}$ we shall apply a variant of the Gram-Schmidt procedure as follows:

(i) For $k>0$ define the polynomials $C_{k+1, k+1} \equiv 2 \cos \theta B_{k k}$ and $S_{k+1, k+1} \equiv$ $2 \sin \theta B_{k k}$. Note that both these polynomials are orthogonal to $B_{k-2, k-2}$ and all earlier members of the sequence. For instance, $\left(C_{k+1, k+1}, B_{k-2, k-2}\right)=\left(2 \cos \theta B_{k k}\right.$, $\left.B_{k-2, k-2}\right)=\left(B_{k k}, 2 \cos \theta B_{k-2, k-2}\right)$, and this is the inner product of $B_{k k}$ with a linear combination of polynomials $B_{k-1, k-1}, B_{k-1, k-2}, \cdots, B_{00}$. This inner product vanishes by hypothesis. For $k=0$ the initialization procedure is described later.

(ii) Orthogonalize $C_{k+1, k+1}$ and $S_{k+1, k+1}$ with respect to each of the polynomials $B_{k-1, k-2}, B_{k-1, k-1}, B_{k, k-1}, B_{k k}$. Call the resulting polynomials $C_{k+1, k+1}^{\prime}, S_{k+1, k+1}^{\prime}$. Specifically,

$$
\begin{aligned}
& C_{k+1, k+1}^{\prime}=C_{k+1, k+1}-\sum B_{r s}\left(C_{k+1, k+1}, B_{r s}\right) /\left(B_{r s}, B_{r s}\right), \\
& S_{k+1, k+1}^{\prime}=S_{k+1, k+1}-\sum B_{r s}\left(S_{k+1, k+1}, B_{r s}\right) /\left(B_{r s}, B_{r s}\right),
\end{aligned}
$$

where $r, s$ take the four value-pairs mentioned.

(iii) Define $B_{k+1, k}$ to be that combination of $C_{k+1, k+1}^{\prime}, S_{k+1, k+1}^{\prime}$ for which the coefficients of $\sin (k+1) \theta$ and $\cos (k+1) \theta$ are one and zero respectively.

(iv) Define $B_{k+1, k+1}$ to be that combination of $C_{k+1, k+1}^{\prime}, S_{k+1, k+1}^{\prime}$ which is orthogonal to $B_{k+1, k}$ and which is normalized to make the coefficient of $\cos (k+1) \theta$ one. If the above algorithm is executable, then clearly it must give rise to polynomials with the desired orthogonality and normalization properties. It remains to examine situations in which the algorithm might not be executable. Stage (ii) could fail if $\left(B_{r s}, B_{r s}\right)=0$. This could only occur if $B_{r s}\left(\theta_{i}\right)=0$ at every data point $\theta_{i}$, i.e., $B_{r s}$ is "degenerate" in the sense mentioned earlier. We may note that under the restriction (which can often be reasonably assumed) that the length of $I^{\prime}$ is less than $2 \pi, B_{k k}$ cannot have more than $2 k$ zeros in $I^{\prime}$. It cannot, therefore, be degenerate unless $2 k \geqq N$, and this would mean that the problem is under-determined, since $B_{k k}$ has $2 k+1$ coefficients. Ordinarily therefore, the nonexecutability of stage (ii) will imply an impror problem formulation. Be that as it may, the algorithm should be terminated vecause the degeneracy of $\boldsymbol{B}_{k k}$ implies the degeneracy of all subsequent functions, so that no further error reduction can be achieved, regardless of how far the space of the approximants may be extended. In order to verify that stage (iii) is executable, we need to find the leading terms, i.e., coefficients of $\sin (k+1) \theta$ and $\cos (k+1) \theta$ in $C_{k+1, k+1}^{\prime}, S_{k+1, k+1}^{\prime}$. Since the leading terms of $B_{k k}$ are $S_{k}, 1$, then those of $C_{k+1, k+1}$ are $s_{k}, 1$ and those of $S_{k+1, k+1}$ are $1,-s_{k}$. Since stage (ii) has no effect on the leading terms, the above value-pairs also hold for $C_{k+1, k+1}^{\prime}$ and $S_{k+1, k+1}^{\prime}$. The required linear combination (iii) is, therefore, $B_{k+1, k}=\left(S_{k+1, k+1}^{\prime}+s_{k} C_{k+1, k+1}^{\prime}\right) /\left(1+s_{k}^{2}\right)$. In stage (iv) both $C_{k+1, k+1}^{\prime}$ and $S_{k+1, k+1}^{\prime}$ are, in principle, subjected to one more orthogonalization process, which will affect the high-order sine coefficients but not the high-order cosine coefficients. Since the high-order cosine coefficient of $C_{k+1, k+1}^{\prime}$ already has the desired value of unity, there is no need to perform the additional orthogonalization on $S_{k+1, k+1}^{\prime}$ and stage (iv) can, therefore, be replaced by:

$$
\text { (iv) } \quad B_{k+1, k+1}=C_{k+1, k+1}^{\prime}-B_{k+1, k}\left(C_{k+1, k+1}^{\prime}, B_{k+1, k}\right) /\left(B_{k+1, k}, B_{k+1, k}\right) \text {. }
$$


The algorithm is initialized by setting $B_{00}=1$, and treating all negative-subscripted functions as identically zero. Furthermore, when $k=0$, step (i) is replaced by $C_{11}=$ $\cos \theta, S_{11}=\sin \theta$. Thereafter, one obtains $B_{10}, B_{11}$ by applying steps (ii), (iii), (iv) with $s_{0}=0$.

Now that the orthogonal functions have been generated, the rest of the curvefitting process will follow the conventional pattern.

In studying the computational cost of the algorithm, it will generally be fair to assume that this is concentrated in the inner products. This is equivalent to assuming that the number of points greatly exceeds the number of free coefficients in the approximant. A formal count of inner products shows twelve in stage (ii) and two in stage (iv)'. However, by taking account of duplications and other relationships, the count can be reduced to seven. All self-inner-products of the form $\left(B_{r r}, B_{r r}\right)$ and $\left(B_{r+1, r}, B_{r+1, r}\right)$ are needed, and these make up two of the seven. Some relations which may be used to economize in stage (ii) are for $k>1$,

$$
\begin{aligned}
& \left(C_{k+1, k+1}, B_{k-1, k-2}\right)=\left(2 \cos \theta B_{k k}, B_{k-1, k-2}\right)=\left(B_{k k}, 2 \cos \theta B_{k-1, k-2}\right)=0, \\
& \left(C_{k+1, k+1}, B_{k-1, k-1}\right)=\left(B_{k k}, 2 \cos \theta B_{k-1, k-2}\right)=\left(B_{k k}, B_{k k}\right), \\
& \left(S_{k+1, k+1}, B_{k-1, k-2}\right)=\left(B_{k k}, 2 \sin \theta B_{k-1, k-2}\right)=-\left(B_{k k}, B_{k k}\right), \\
& \left(S_{k+1, k+1}, B_{k-1, k-1}\right)=\left(B_{k k}, 2 \sin \theta B_{k-1, k-1}\right)=-s_{k-1}\left(B_{k k}, B_{k k}\right) .
\end{aligned}
$$

This leaves five more inner products to be explicitly computed, four for stage (ii) and the last for stage (iv)'. These are:

$$
\begin{aligned}
\left(C_{k k}, B_{k, k-1}\right) & =2\left(\cos \theta B_{k k}, B_{k, k-1}\right),\left(C_{k k}, B_{k k}\right)=2\left(\cos \theta B_{k k}, B_{k k}\right), \\
\left(S_{k k}, B_{k, k-1}\right) & =2\left(\sin \theta B_{k k}, B_{k, k-1}\right),\left(S_{k k}, B_{k k}\right)=2\left(\sin \theta B_{k k}, B_{k k}\right), \\
\left(C_{k+1, k+1}^{\prime}, B_{k+1, k}\right) & =2\left(\cos \theta B_{k k}, B_{k+1, k}\right) .
\end{aligned}
$$

If the computational cost of constructing the orthogonal functions is truly reflected by the number of inner products, then our average cost is $3 \frac{1}{2}$ inner products for each single augmentation of the set of orthogonal functions. The comparative cost for generating orthogonal algebraic polynomials is 3 inner products per augmentation. Since, in each case, it can ordinarily be assumed that at least one Fourier coefficient will be required corresponding to each orthogonal function, the cost ratio will ordinarily be no worse than $4 \frac{1}{2}: 4$. In principle, one can also build on ordinate and derivative constraints to this algorithm as was done in the case of the pure sine and cosine polynomials, but the details of this will not be discussed here.

Experimental Results. In order to test the capabilities of the above algorithm for least-squares approximation by balanced trigonometric polynomials, the following experiment was run:

(A) Define $f(x)=1+|\sin x|+|\cos 2 x|$.

(B) Define 60 equal spaced abscissas in the interval $(0,3 \pi / 2)$ and delete 10 of them randomly. Call this set of abscissas $X$.

(C) Let a number $\rho$ take consecutively the five values $.2, .4, \cdots, 1$. For each $\rho$ value generate 10 sets of 50 random numbers uniformly distributed in $(-\rho, \rho)$. Add the 50 random numbers to the 50 evaluations of $f(x)$ on $X$. Thus, for each $\rho$ we have ten "noised-up" data sets generated by $f(x)$. 
(D) Since the period of $f(x)$ is $\pi$, we infer that the "best" value of $\omega$ in the equalweighted least-squares sense should be around 2 . We wish to determine how far this fact may be verified by the algorithm in the presence of increasing noise. For each "noised-up" data set we try five values of $\omega$, namely, $2 \pm 5 \%$ and $2 \pm 10 \%$, and we observe how often it happens that the value $\omega=2$ is best of the five in the least-squares sense. The approximant went as far as terms involving $2 \theta$. The results are given in the table below, which indicates the ranking of the value $\omega=2$ for the various $\rho$-values. For example, when $\rho=.6$ the value $\omega=2$ was the best of the five $\omega$-values on seven of the ten "noised-up" data sets.

\begin{tabular}{cccccc} 
& 1st & 2nd & 3rd & 4th & 5th \\
\hline$\rho=.2$ & 10 & 0 & 0 & 0 & 0 \\
.4 & 8 & 2 & 0 & 0 & 0 \\
.6 & 7 & 1 & 2 & 0 & 0 \\
.8 & 5 & 3 & 2 & 0 & 0 \\
1. & 4 & 3 & 3 & 0 & 0 \\
\hline
\end{tabular}

From these figures it is seen that the $\pi$-periodicity is still detectable, though not very strongly, even at the highest noise level of $\rho=1$. It should be noted that in two respects the test may be considered as fairly stringent; firstly, the undifferentiable function $f(x)$ is not readily approximated by a low-order Fourier series, and secondly, the data were spread over only one-and-a-half periods. Thus, there was only one-half of a period during which any evidence of periodicity could be gathered. In view of these inherent difficulties and of the noise level imposed, it would seem that this algorithm has quite a high potential for detecting harmonic periodicities against a background of noise.

In conclusion, it should be observed that in all the algorithms discussed above it is possible, with minor changes, to read 'sinh, cosh' for 'sine, cos' and thus to produce corresponding algorithms for interpolation and approximation in terms of hyperbolic functions.

University of Kentucky

Lexington, Kentucky 40506

1. A. C. R. NewBery, "Interpolation by algebraic and trigonometric polynomials," Math. Comp., v. 20, 1966, pp. 597-599. MR 34 \#3752.

2. P. J. DAvIs, Interpolation and Approximation, Blaisdell, Waltham, Mass., 1963. MR 28 \#393.

3. F. B. HiLdebrand, Introduction to Numerical Analysis, McGraw-Hill, New York, 1956. MR 17, 788 .

4. F. Oliveira-Pinto, "Curve-fitting to unequally-spaced data: Polynomial and trigonometric approximation," Inst. Gulbenkian Ci. Centro Calc. Ci. Estud. Program. Anál. Numér., No. 2, 1967, pp. 47-59. MR $37 \# 2408$.

5. G. E. FORSYTHE, "Generation and use of orthogonal polynomials for data fitting with a digital computer," J. Soc. Indust. Appl. Math., v. 5, 1957, pp. 74-88. MR 19, 1079.

6. R. W. KLOPFENSTEIN, "Conditional least squares polynomial approximation," Math. Comp., v. 18, 1964, pp. 659-662. MR $29 \# 6611$. 